\title{
SHEA neonatal intensive care unit (NICU) white paper series: Practical approaches to Clostridioides difficile prevention
}

\author{
Thomas J. Sandora MD, MPH${ }^{1}$, Kristina K. Bryant MD², Joseph B. Cantey MD ${ }^{3}$, Alexis M. Elward MD, MPH ${ }^{4}$, \\ Deborah S. Yokoe MD, MPH ${ }^{5}$ and Allison H. Bartlett MD, $\mathrm{MS}^{6}$ \\ ${ }^{1}$ Boston Children's Hospital, Boston, Massachusetts, ${ }^{2}$ University of Louisville, Louisville, Kentucky, ${ }^{3}$ The University of Texas Health Science Center, San Antonio, \\ Texas, ${ }^{4}$ Washington University School of Medicine, St Louis, Missouri, ${ }^{5}$ University of California, San Francisco, California and ${ }^{6}$ University of Chicago and Comer \\ Children's Hospital, Chicago, Illinois
}

(Received 17 July 2018; accepted 21 July 2018; electronically published August 30, 2018)

\section{Introduction}

This white paper provides clinicians with practical advice related to Clostridioides difficile in neonatal intensive care unit (NICU) patients. Clostridioides difficile infection (CDI) is among the most prevalent and important healthcare-associated infections (HAIs) affecting children, ${ }^{1,2}$ but reports of CDI in infants $<12$ months of age are rare, perhaps because of a relative resistance to the effects of C. difficile toxins or other protective factors in the intestinal environment of infants. ${ }^{3}$ Additionally, high colonization rates in infants ( $35 \%)$ make interpretation of positive $C$. difficile tests in NICU patients uniquely challenging. For these reasons, the authors do not recommend routine testing for $C$. difficile in NICU patients; NICU patients should be evaluated for other more common causes of diarrhea. Few data exist in the published literature about preventing $C$. difficile in the NICU in endemic settings.

\section{Intended use}

The SHEA intends this document to serve as a companion to the Centers for Disease Control and Prevention (CDC) Healthcare Infection Control Practices Advisory Committee (HICPAC) "Clostridioides difficile in Neonatal Intensive Care Unit Patients: A Systematic Review," to provide practical, expert opinion-based answers to frequently asked questions on $C$. difficile detection and prevention in the NICU. These questions are not adequately addressed in the published literature to meet Grading of Recommendations Assessment, Development and Evaluation (GRADE) standards ${ }^{4}$ and therefore were not included in the HICPAC systematic review.

No guideline, expert guidance, or white paper can anticipate all situations, and this document is not meant to be a substitute for individual judgment by qualified professionals.

\footnotetext{
Author for correspondence: Thomas J. Sandora, MD, MPH, Boston Children's Hospital, 300 Longwood Avenue, Boston, MA 02115. E-mail: thomas.sandora@childrens. harvard.edu

Cite this article: Sandora TJ, et al. (2018). SHEA neonatal intensive care unit (NICU) white paper series: Practical approaches to Clostridioides difficile prevention. Infection Control \& Hospital Epidemiology 2018, 39, 1149-1153. doi: 10.1017/ice.2018.209

\section{Methods}

This document was developed by a writing panel of pediatric and pathogen-specific experts who collaborated with members of the HICPAC systematic review writing panel and the SHEA Pediatric Leadership Council to identify questions that should be addressed. Unlike the SHEA expert guidance format, this document is not based on a systematic literature search; instead, for the selected topic, the authors provide practical approaches in question-and-answer format based on expert opinion and consensus within the context of the HICPAC systematic review.

The SHEA Guidelines Committee and the SHEA Publications Committee reviewed this document, and the SHEA Board of Trustees endorsed it. The paper has also been endorsed by the American Hospital Association (AHA), The Joint Commission, The Pediatric Infectious Diseases Society (PIDS), the Association for Professionals in Infection Control and Epidemiology (APIC), the Infectious Diseases Society of America (IDSA), and the National Association of Neonatal Nurses (NANN).

\section{Authors}

The authors are current and past members of the SHEA Guidelines Committee and the SHEA Pediatric Leadership Council, who serve as volunteers. All authors are involved at their respective institutions in the development of policies pertaining to pediatric infection prevention, either directly or in an advisory role.

The NICU Advisory Panel, which provided oversight and review of the paper, is composed of representatives from the Society for Healthcare Epidemiology of America (SHEA), the American Academy of Pediatrics (AAP), the Association for Professionals in Infection Control and Epidemiology (APIC), the Infectious Diseases Society of America (IDSA), the National Association of Neonatal Nurses (NANN), the Pediatric Infectious Diseases Society (PIDS), and The Joint Commission.

\section{Practical approaches: questions and answers}

Question: When should clinicians test a NICU patient for CDI? Answer:

- The authors advise against routine testing for CDI in NICU patients because of the high prevalence of asymptomatic 
carriage of toxigenic $C$. difficile in infants $<12$ months of age. When $C$. difficile or its toxins are detected in the stool of an infant, clinicians may not be able to determine with certainty that a positive result represents CDI.

- Clinicians should test NICU patients for CDI only if there is evidence of pseudomembranous colitis or if the patient has clinically significant diarrhea and other noninfectious and infectious causes of diarrhea have been excluded.

In general, a case of CDI is defined as the presence of clinically significant diarrhea or toxic megacolon in conjunction with either a positive test for toxigenic $C$. difficile or its toxins in the stool, or evidence of pseudomembranous colitis by endoscopy, surgery, or histopathology. For adults, the IDSA/SHEA clinical practice guideline for $C$. difficile infection recommends patients with unexplained and new-onset $\geq 3$ unformed stools in 24 hours as the preferred target population for testing for CDI. ${ }^{5}$ Testing patients without clinically significant diarrhea will decrease the positive predictive value of a positive test for $\mathrm{CDI}^{6}{ }^{6}$ but no universally accepted or validated definition of clinically significant diarrhea exists for infants. In addition, infants $<12$ months of age have a high rate of colonization with C. difficile, ${ }^{7-9}$ and even when diarrhea is present, it is difficult to be certain that a positive test result represents CDI (as opposed to the detection of colonization in a patient in whom there may be alternative etiologies of diarrhea). For these reasons, and because of the lack of a clear association between positive tests and true clinical disease in infants, the IDSA/SHEA guideline recommends that testing for CDI should never be routinely recommended in endemic circumstances for neonates or infants $<12$ months of age who have diarrhea. ${ }^{5}$

Question: Before or concurrent with C. difficile testing, which gastrointestinal pathogens should clinicians test for in a NICU patient who develops diarrhea?

Answer: Before testing a NICU patient with suspected infectious diarrhea for CDI, clinicians should

- Perform a thorough investigation for potential noninfectious causes of diarrhea in NICU patients.

- Test the stool for norovirus, rotavirus, adenovirus, and enterovirus.

- Consider bacterial stool cultures (eg, Salmonella, Shigella, Campylobacter, Yersinia, and Shiga-toxin-producing E. coli) for infants who were admitted to the NICU from the community, or who have a known or suspected exposure to bacterial enteritis.

Approximately $35 \%$ of neonates are colonized with C. difficile, and toxigenic strains can persist for months. ${ }^{3,8,10}$ In the absence of a clear association with infant disease, the authors do not advise testing for CDI unless other possibilities have been excluded. ${ }^{11}$

Because diarrhea in a NICU patient is often noninfectious, before testing for CDI, clinicians should perform a thorough examination and review of the infant's medical history, feeding, and medications to exclude other more likely causes of diarrhea (eg, feeding intolerance or milk protein allergy, malabsorption, opioid withdrawal, etc).

Cohort studies evaluating the prevalence of viruses in the stool of NICU patients have shown low endemic rates of gastrointestinal pathogens. ${ }^{12}$ Norovirus, rotavirus, enteroviruses, and adenoviruses are common pathogens and have been reported to cause infections and outbreaks in preterm infants. ${ }^{13-17}$ Therefore, the authors suggest testing for these viruses in an infant with suspected infectious diarrhea if providers have access to a laboratory with those capabilities. The presence of $C$. difficile toxin can create cytopathic effects in tissue culture and can lead to false-positive results for stool viral culture. ${ }^{18}$ Because $C$. difficile toxin would be expected to be present due to colonization in a substantial proportion of infants, the authors suggest that facilities use polymerase chain reaction (PCR) assays (a sensitive and rapid testing method for these viruses) as a preferred modality for viral testing. An outbreak may warrant a broader investigation of potential pathogens.

Bacterial pathogens such as Salmonella ${ }^{19}$ and Escherichia coli ${ }^{20-22}$ have been linked to NICU outbreaks. Routinely testing NICU patients with suspected infectious diarrhea for bacterial pathogens is unlikely to have a high yield, but in the setting of a community or hospital outbreak, symptomatic family or healthcare contacts, recent admission from the community, or concern for bloody or mucopurulent diarrhea, such testing may be warranted. Some institutions use multiplex PCR assays to identify gastrointestinal pathogens, and some of these panels include $C$. difficile. If a multiplex panel is used to test a NICU patient for other pathogens, clinicians should be aware that $C$. difficile may be detected with high frequency in this age group. A positive result may reflect colonization rather than infection and may not warrant treatment. ${ }^{23}$ For this reason, some facilities suppress the $C$. difficile result on these panels and require a separate dedicated test if CDI is a concern.

Question: What are the best methods for testing for CDI in NICU patients?

Answer:

- If clinicians consider testing a NICU patient for CDI, the authors advise using a stool toxin test as part of a multistep algorithm rather than using a nucleic acid amplification test (NAAT) alone.

- The facility should not use toxin enzyme immunoassay (EIA) as a stand-alone test to diagnose CDI.

- Repeat testing after a negative result and tests of cure are not recommended.

Because CDI is a toxin-mediated disease, any testing strategy must include detection of either toxin or a toxigenic organism.

1. NAATs, primarily polymerase chain reaction testing for the genes for toxins $\mathrm{A}$ and $\mathrm{B}$,

- Are more sensitive for $C$. difficile detection than toxin EIA tests, but

- Their positive predictive value can be low, ${ }^{24}$ particularly when the prevalence of colonization is high (as is true for infants).

\section{Toxin EIA testing}

- In addition to having lower sensitivity than NAAT, may also be prone to false-positive results in children. ${ }^{25}$

- Is not recommended as a stand-alone approach to diagnosis.

3. Glutamate dehydrogenase (GDH) immunoassays

- Detect a highly conserved antigen present in all $C$. difficile isolates.

- Can be used as a component of 2- or 3-step algorithms with subsequent toxin testing, in which a negative toxin EIA result is sometimes arbitrated by NAAT as outlined in the IDSA/SHEA guideline. ${ }^{5}$ 
In a population with a low pretest probability of disease, such as NICU patients, the authors believe that it is most appropriate to choose a diagnostic approach that includes a toxin test to increase the specificity of the result. Although some experts have recommended that toxigenic culture be included as part of CDI testing for infants, ${ }^{26}$ it is impractical for most clinical labs to perform culture and this approach is rarely used in practice.

Repeat testing after an initial negative result is associated with higher rates of false-positive results and unnecessary antibiotic therapy. ${ }^{27-30}$ Similarly, "test of cure" specimens are not useful. C. difficile toxin can persist in stool for up to a month after resolution of diarrhea following treatment. Continued detection of toxin after disease resolution may lead to false-positive test results, unnecessary therapy, and prolonged contact precautions. ${ }^{31,32}$

Question: What is the preferred hand hygiene agent when caring for NICU patients who test positive for C. difficile?

Answer:

1. In a nonoutbreak setting, there is no consensus on the optimal approach to hand hygiene when caring for a patient with CDI. Based on a hospital's assessment of risk, any of the following options could be considered:

- Standard hand hygiene using alcohol-based hand rub (ABHR) for room entry and exit

- Soap and water hand hygiene for room exit only, with $\mathrm{ABHR}$ for room entry and when needed between tasks for a single patient unless hands are visibly soiled

- Soap and water preferred over ABHR for room entry and exit

2. Soap and water are recommended for hand hygiene during a $C$. difficile outbreak or in hyperendemic settings.

3. The facility must consider sink accessibility when making recommendations for soap-and-water hand hygiene.

Barriers to hand hygiene will have the effect of decreasing hand hygiene compliance, so hand hygiene should be made as convenient as possible. Gloves are effective at preventing contamination of the hands with $C$. difficile, but they are not a substitute for effective hand hygiene. ${ }^{31}$ Patients may be infected or colonized with $>1$ pathogen, and $A B H R$ effectively interrupts transmission of most other organisms. ${ }^{33}$ It is reasonable to use ABHR before patient contact and within the patient's area when moving from dirty to clean (eg, after a dressing change or diaper change, before starting a feeding).

Although alcohol is not sporicidal, handwashing with soap and water is not a perfect option, as handwashing may remove $<1 \log _{10}$ of $C$. difficile spores. ${ }^{31}$ The potential advantage of soap and water for $C$. difficile spores must be weighed against the potential disadvantage of lower hand hygiene compliance when soap and water use is recommended. Hospitals should make recommendations according to their assessment of risk, as well as the availability of infrastructure (sinks and ABHR) to support those recommendations.

Question: What is the appropriate type and duration of isolation for an infant with a positive $C$. difficile test?

\section{Recommendations:}

- Infants with diarrhea and a positive $C$. difficile test should be placed on contact precautions. Infants without diarrhea who have tested positive for $C$. difficile do not require contact precautions.
- Contact precautions can be discontinued 48 hours after diarrhea has resolved. At that time, consideration can be given to moving the patient to a new incubator and/or a new room.

Clostridioides difficile infections are transmitted from infected patients to other patients, either directly or indirectly, through the environment. Hands of healthcare workers in conjunction with environmental contamination are recognized sources of transmission. ${ }^{34,35}$ Healthcare worker hand contamination increases as the rate of environmental contamination increases. Environmental contamination is higher in rooms of patients with C. difficile-associated diarrhea than in rooms of patients who do not have diarrhea. ${ }^{36}$ Patients with asymptomatic $C$. difficile colonization shed spores, but generally they shed fewer spores and cause less contamination than symptomatic patients. ${ }^{31}$ Thus, contact precautions are important to preventing transmission from patients with CDI and should be used until 48 hours after resolution of diarrhea. Although prolongation of contact precautions until discharge is a strategy that can be adopted for older patients when CDI rates remain high, extending this practice to NICU patients is not recommended for the following reasons:

1. Determining true CDI rates in the NICU is difficult because testing is not recommended and positive results most likely reflect colonization.

2. Prolonging contact precautions until discharge could result in extended isolation for premature infants who are expected to remain hospitalized for long durations. Although emerging data suggest that asymptomatic carriers may also play a role in transmission, current guidelines do not recommend using contact precautions for colonized patients without diarrhea. ${ }^{5}$

Bacterial burden in stool of patients with CDI decreases with appropriate treatment, but it does not correlate with the amount of diarrhea. ${ }^{37}$ Given the risk of persistent contamination of environmental surfaces in a patient's room (and inside and around an incubator), consideration should be given to moving the patient to a new room and/or changing the incubator or warmer after diarrhea has resolved to further decrease the risk of transmission, although no specific data addressing the effectiveness of this strategy are available.

Question: What is the appropriate cleaning and disinfection strategy for C. difficile in the NICU?

Answer:

- In endemic settings, standard daily cleaning is appropriate.

- Bleach or another product with an Environmental Protection Agency-approved claim for $C$. difficile sporicidal activity should be considered for disinfecting the environment during CDI outbreaks or in hyperendemic settings.

- For patients whose CDI has resolved but whose continued hospitalization is required, consider moving the patient to a new room once diarrhea has resolved.

- Incubators may be thought of similarly to the patient's room:

- In endemic settings, standard cleaning and processing of an incubator should occur before it is used for the next patient.

- Consider moving the patient to a clean incubator when diarrhea has resolved. 
- Develop a strong communication plan with environmental services to identify rooms requiring cleaning with a sporicidal disinfectant.

Data are conflicting as to whether inactivation of spores is necessary to prevent $C$. difficile transmission in the endemic setting. ${ }^{31}$ The decision to use a sporicidal disinfectant (eg, bleach) for $C$. difficile patient rooms in a nonoutbreak setting should take into consideration workflow and communication with environmental services. A system will be needed to alert environmental services staff to rooms requiring sporicidal disinfection.

No studies have examined incubators as potential reservoirs for C. difficile, though incubators have been implicated in transmission of vancomycin-resistant Enterococcus from patient to patient in a published study. ${ }^{38}$

Question: What is the role for antimicrobial stewardship in the prevention of $C$. difficile infection in infants?

Answer: Antimicrobial stewardship has resulted in decreases in CDI in many populations, and because of its beneficial impact on other adverse events seen in the NICU, encouraging appropriate use of antimicrobials in the NICU should be a priority.

Antimicrobial stewardship efforts have been shown to significantly decrease rates of CDI in adults, ${ }^{39}$ although data for the neonatal population are lacking and would be more challenging to interpret given high colonization rates. Prolonged use of empiric antibiotics in neonates has been associated with an increased risk of necrotizing enterocolitis and death ${ }^{40}$ and invasive candidiasis. ${ }^{41}$ Therefore, although no specific recommendation can be made regarding antimicrobial stewardship as a means of prevention of CDI in the NICU, every NICU should have stewardship processes in place to ensure appropriate use of antimicrobials.

Acknowledgments. The authors thank Susan Coffin, MD, MPH at Children's Hospital of Philadelphia (CHOP) for her extensive and thoughtful review of the manuscript. NICU Advisory Panel: Kenneth M. Zangwill, MD, American Academy of Pediatrics (AAP); Nancy Foster, American Hospital Association (AHA); Katrina Crist, MBA, CAE, Association for Professionals in Infection Control and Epidemiology (APIC); Beth Huizinga, MSN, RNC-NIC, National Association of Neonatal Nurses (NANN); Pablo Sanchez, MD, Infectious Diseases Society of America (IDSA); Aaron M. Milstone, MD, Pediatric Infectious Diseases Society (PIDS); Margaret VanAmringe, MHS, The Joint Commission.

Conflicts of Interest. The following disclosures have been reported to SHEA. To provide thorough transparency, SHEA requires full disclosure of all relationships, regardless of relevancy to the guideline topic. Evaluation of such relationships as potential conflicts of interest is determined by a review process. The assessment of disclosed relationships for possible COI will be based on the relative weight of the financial relationship (ie, monetary amount) and the relevance of the relationship (ie, the degree to which an association might reasonably be interpreted by an independent observer as related to the topic or recommendation of consideration). The reader of this guidance should be mindful of this when the list of disclosures is reviewed.

T.S. reports research grants/contracts with NIH/NIAID BIDMC/Boston Children's Hospital: Ultrasensitive C. difficile Toxin Measurement for Diagnosis and Outcome Prediction; LEAP (IDSA, SHEA, PIDS): assessing and optimizing the utility of the Massachusetts statewide antibiogram; hospital bacterial genomics to elucidate the source of HAIs; effect of a revised clinical practice guideline for empiric treatment of fever and neutropenia on microbiological resistance patterns in pediatric oncology patients. K.B. reports honoraria from MedStudy, research funding from Pfizer for clinical vaccine trials, clinical funding from Kosair Charities for the University of Louisville Department of Pediatrics Fellowship Program in Pediatric Infectious Disease $(>\$ 25,000)$, and organizational involvement with PIDS and the PIDS Foundation. A.B. reports advisory/consultant role with CVS/Caremark, research grants/contracts with
NIDCD Lead Investigator: Albert H. Park, MD, University of Utah School of Medicine, 'Randomized Controlled Trial of Valganciclovir for Asymptomatic Cytomegalovirus Infected Hearing Impaired Infants' PCORI Lead Investigator: Jane C. Burns, MD, UCSD, Kawasaki Disease Comparative Effectiveness Trial. All other authors report that they have nothing to disclose.

\section{References}

1. Sammons JS, Toltzis P. Recent trends in the epidemiology and treatment of C. difficile infection in children. Curr Opin Pediatr 2013;25:116-121.

2. Wendt JM, Cohen JA, Mu Y, et al. Clostridium difficile infection among children across diverse US geographic locations. Pediatrics 2014;133:651-658.

3. Jangi S, Lamont JT. Asymptomatic colonization by Clostridium difficile in infants: implications for disease in later life. J Pediatr Gastroenterol Nutr 2010;51:2-7.

4. Guyatt GH, Oxman AD, Vist GE, et al. GRADE: an emerging consensus on rating quality of evidence and strength of recommendations. BMJ 2008;336:924-926.

5. McDonald LC, Gerding DN, Johnson S, et al. Clinical practice guidelines for Clostridium difficile infection in adults and children: 2017 update by the Infectious Diseases Society of America (IDSA) and Society for Healthcare Epidemiology of America (SHEA). Clin Infect Dis 2018;66:e1-e48.

6. Dubberke ER, Han Z, Bobo L, et al. Impact of clinical symptoms on interpretation of diagnostic assays for Clostridium difficile infections. $J$ Clin Microbiol 2011;49:2887-2893.

7. Sherertz RJ, Sarubbi FA. The prevalence of Clostridium difficile and toxin in a nursery population: a comparison between patients with necrotizing enterocolitis and an asymptomatic group. J Pediatr 1982;100:435-439.

8. Rousseau C, Lemee L, Le Monnier A, Poilane I, Pons JL, Collignon A. Prevalence and diversity of Clostridium difficile strains in infants. J Med Microbiol 2011;60:1112-1118.

9. Tamma PD, Sandora TJ. Clostridium difficile infection in children: current state and unanswered questions. J Pediatric Infect Dis Soc 2012;1:230-243.

10. Adlerberth I, Huang H, Lindberg E, et al. Toxin-producing Clostridium difficile strains as long-term gut colonizers in healthy infants. J Clin Microbiol 2014;52:173-179.

11. Schutze GE, Willoughby RE. Clostridium difficile infection in infants and children. Pediatrics 2013;131:196-200.

12. Naing Z, Rayner B, Killikulangara A, et al. Prevalence of viruses in stool of premature neonates at a neonatal intensive care unit. J Paediatr Child Health 2013;49:E221-E226.

13. Shim JO, Son DW, Shim SY, Ryoo E, Kim W, Jung YC. Clinical characteristics and genotypes of rotaviruses in a neonatal intensive care unit. Pediatr Neonatol 2012;53:18-23.

14. Stuart RL, Tan K, Mahar JE, et al. An outbreak of necrotizing enterocolitis associated with norovirus genotype GII.3. Pediatr Infect Dis J 2010;29:644-647.

15. Turcios-Ruiz RM, Axelrod P, St John K, et al. Outbreak of necrotizing enterocolitis caused by norovirus in a neonatal intensive care unit. $J$ Pediatr 2008;153:339-344.

16. Kusuhara K, Saito M, Sasaki Y, et al. An echovirus type 18 outbreak in a neonatal intensive care unit. Eur J Pediatr 2008;167:587-589.

17. Finn A, Anday E, Talbot GH. An epidemic of adenovirus 7a infection in a neonatal nursery: course, morbidity, and management. Infect Control Hosp Epidemiol 1988;9:398-404.

18. Faden H, Patel PH, Campagna L. Pitfalls in the diagnosis of enteroviral infection in young children. Pediatr Infect Dis J 2006;25:687-690.

19. Boehmer TK, Bamberg WM, Ghosh TS, et al. Health care-associated outbreak of Salmonella Tennessee in a neonatal intensive care unit. Am J Infect Control 2009;37:49-55.

20. Taneja N, Das A, Raman Rao DS, Jain N, Singh M, Sharma M. Nosocomial outbreak of diarrhoea by enterotoxigenic Escherichia coli among preterm neonates in a tertiary care hospital in India: pitfalls in healthcare. J Hosp Infect 2003;53:193-197.

21. Gross RJ, Rowe B, Henderson A, Byatt ME, Maclaurin JC. A new Escherichia coli O-group, O159, associated with outbreaks of enteritis in infants. Scand J Infect Dis 1976;8:195-198. 
22. Gaynes RP, Simpson D, Reeves SA, et al. A nursery outbreak of multipleaminoglycoside-resistant Escherichia coli. Infect Control 1984;5:519-524.

23. Buss SN, Leber A, Chapin K, et al. Multicenter evaluation of the BioFire FilmArray gastrointestinal panel for etiologic diagnosis of infectious gastroenteritis. J Clin Microbiol 2015;53:915-925.

24. Planche TD, Davies KA, Coen PG, et al. Differences in outcome according to Clostridium difficile testing method: a prospective multicentre diagnostic validation study of C. difficile infection. Lancet Infect Dis 2013;13:936-945.

25. Toltzis P, Nerandzic MM, Saade E, et al. High proportion of false-positive Clostridium difficile enzyme immunoassays for toxin A and B in pediatric patients. Infect Control Hosp Epidemiol 2012;33:175-179.

26. Bryant $\mathrm{K}$, McDonald LC. Clostridium difficile infections in children. Pediatr Infect Dis J 2009;28:145-146.

27. Mohan SS, McDermott BP, Parchuri S, Cunha BA. Lack of value of repeat stool testing for Clostridium difficile toxin. Am J Med 2006;119:356 e7-e8.

28. Luo RF, Banaei N. Is repeat PCR needed for diagnosis of Clostridium difficile infection? J Clin Microbiol 2010;48:3738-3741.

29. Aichinger E, Schleck CD, Harmsen WS, Nyre LM, Patel R. Nonutility of repeat laboratory testing for detection of Clostridium difficile by use of PCR or enzyme immunoassay. J Clin Microbiol 2008;46:3795-3797.

30. Peterson LR, Robicsek A. Does my patient have Clostridium difficile infection? Ann Intern Med 2009;151:176-179.

31. Dubberke ER, Carling P, Carrico R, et al. Strategies to prevent Clostridium difficile infections in acute care hospitals: 2014 update. Infect Control Hosp Epidemiol 2014;35:628-645.

32. Surawicz CM, Brandt LJ, Binion DG, et al. Guidelines for diagnosis, treatment, and prevention of Clostridium difficile infections. Am J Gastroenterol 2013;108:478-498.
33. WHO guidelines on hand hygiene in health care. World Health Organization website. http://www.who.int/gpsc/5may/tools/9789241597906/ en/. Published 2009. Accessed July 31, 2018.

34. McFarland LV, Mulligan ME, Kwok RY, Stamm WE. Nosocomial acquisition of Clostridium difficile infection. N Engl J Med 1989;320:204-210.

35. Samore MH, Venkataraman L, DeGirolami PC, Arbeit RD, Karchmer AW. Clinical and molecular epidemiology of sporadic and clustered cases of nosocomial Clostridium difficile diarrhea. Am J Med 1996;100:32-40.

36. Dubberke E, Reske K, Noble-Wang J, et al. Prevalence of Clostridium difficile environmental contamination and strain variability in multiple health care facilities. Am J Infect Control 2007;35:315-318.

37. El Feghaly R, Stauber J, Deych E, Gonzalez C, Tarr P, Haslam D. Markers of intestinal inflammation, not bacterial burden, correlate with clinical outcomes in Clostridium difficile infection. Clin Infect Dis 2013;56:1713-1721.

38. Golan Y, Doron S, Sullivan B, Snydman DR. Transmission of vancomycin-resistant Enterococcus in a neonatal intensive care unit. Pediatr Infect Dis J 2005;24:566-567.

39. Fowler S, Webber A, Cooper BS, et al. Successful use of feedback to improve antibiotic prescribing and reduce Clostridium difficile infection: a controlled interrupted time series. J Antimicrob Chemother 2007;59:990-995.

40. Cotten CM, Taylor S, Stoll B, et al. Prolonged duration of initial empirical antibiotic treatment is associated with increased rates of necrotizing enterocolitis and death for extremely low birth weight infants. Pediatrics 2009;123:58-66.

41. Cotten CM, McDonald S, Stoll B, Goldberg RN, Poole K, Benjamin DK Jr. The association of third-generation cephalosporin use and invasive candidiasis in extremely low birth-weight infants. Pediatrics 2006;118:717-722. 